Penalva, A., Hernández, M.A. \& Guerrero, C. (2013). La gestión eficaz del docente en el aula. Un estudio de caso. Revista Electrónica Interuniversitaria de Formación del Profesorado, 16 (2), 77-91.

DOI: http://dx.doi.org/10.6018/reifop.16.2.180931

\title{
La gestión eficaz del docente en el aula. Un estudio de caso
}

\author{
Antonia Penalva López, Mángeles Hernández Prados, Catalina Guerrero Romera \\ Universidad de Murcia
}

\section{Resumen}

La calidad educativa es uno de los propósitos de nuestro sistema educativo, lidiado por conseguir escuelas eficaces donde la figura del docente adquiera el papel que le corresponde en la gestión del aula y en la mejora educativa. En esta investigación se analiza la gestión docente de noveles y expertos, en grupos y aulas de similares características, a través de dos instrumentos: uno de observación directa y otro indirecta. Tras el tratamiento estadístico de los datos se han comprobado tres de las hipótesis planteadas, dos fueron rechazadas y una parcialmente confirmada.

\section{Palabras clave}

Gestión de aula; escuelas eficaces; profesor novel; profesor experto.

\section{Effective classroom management. A case study}

\footnotetext{
Abstract

The quality of Education is one of the goals of our education system along with the achievement of effective schools where the teacher assumes a suitable role as classroom manager and teaching optimizer. This research analyses classroom management in trainee and expert teachers, in similar

Contacto

Antonia Penalva López, antonia.penalva@gmail.com, Avd. Ramón y Cajal n $83 \mathrm{P}^{\circ} 2$ izq. Apartado de correos 88, Código Postal: 30530, Cieza (Murcia).

Artículo vinculado a la tesis fin de Máster de investigación e innovación en educación infantil y primaria.
} 
groups and classrooms, by means of two instruments, namely, a direct observation and an indirect observation tool. Statistic data have checked three hypotheses out of the initially established ones; two were rejected and one of them partially confirmed.

\section{Key words}

Classroom managemen; efficacious schools; new teachers; expert teacher

\section{Introducción}

A lo largo de los años la profesión docente se ha visto envuelta en continuos y cambios, producto de las aceleradas transformaciones que se produjeron en las esferas económicas, políticas y sociales que inevitablemente afectaron al sistema educativo y a la labor docente (Martínez, Collazo, \& Liss, 2009). A pesar de los esfuerzos pedagógicos por promover un cambio cualitativo de envergadura, la observación de la práctica docente evidencia que aún queda un largo camino por recorrer. En los centros escolares continúan primando el currículo y los conocimientos, a la transmisión de valores. Por otra parte, son los mismos docentes los que manifiestan cierto malestar en su función educativa, ya que como recoge Jaap Scheerens (1992, p.13, citado en Murillo, 2008) "poseemos algunos conocimientos acerca de qué funciona en educación, pero aún tenemos poca consistencia". Todos estos esfuerzos por el cambio pedagógico y la mejora de la calidad educativa, se remontan a los años 80 , donde el concepto de escuelas eficaces empieza a cobrar importancia y emergen las primeras investigaciones, con la intención de construir modelos teóricos que sirvan de apoyo a la praxis docente. Pero ¿qué se entiende por escuela eficaz?, Es aquella que promueve que cada uno de los alumnos alcance, de forma duradera, el mayor nivel posible en todos los aspectos de su rendimiento y desarrollo integral, más allá de lo que sería previsible teniendo en cuenta su rendimiento inicial y su situación social, cultural y económica (Murillo, 2005). Existen distintos modelos teóricos que proponen diversos factores que caracterizan a una escuela eficaz con el fin de promover el cambio (Ortega, 2008). A continuación, se exponen algunos de los factores más relevantes (Tabla1).

En definitiva, las escuelas eficaces tienen como objetivo, incrementar y garantizar la calidad de los procesos de enseñanza-aprendizaje y de las instituciones a través del trabajo en equipo y colaborativo, de la cultura de liderazgo, de la creación de un clima escolar en el que imperen los valores de la ciudadanía democrática, de la cooperación entre familia y escuela, entre otros aspectos, teniendo en cuenta variables como: el nivel de expectativas de la comunidad escolar, la formación del profesorado, la gestión de proyectos, establecimiento de objetivos claros y perspicaces, etc. (Fullan, 1996; Beltran, 2007; Lavín, 2007; González, 2005; Castro, 2012; González, Vázquez \& Rodríguez, 2013).

Con la intención de conocer en qué medida los centros escolares son eficaces, se han diseñado sistemas de medición de la calidad y del rendimiento de los estudiantes, que tienden a manifestar una baja y desigual calidad de la educación (Gómez, 2010). De igual modo, los resultados del informe PISA (2012), evidencian que los alumnos españoles presentan niveles más bajos de rendimiento de la lectura, matemáticas y ciencias que la media europea. Esta preocupación por la calidad educativa no es algo novedoso, ya en los años 90 ese recoge en la LOGSE en su Título IV la importancia de asegurar la calidad de la enseñanza como uno de los retos de la educación, y la evaluación como uno de los pilares de la misma. Pero coincidimos con Murillo (2003), en la necesidad de una visión más amplia de los conceptos de calidad y gestión, que resalte un papel más dinámico y activo los 
docentes dentro los centros escolares. En definitiva, se trata de considerar la calidad de la actividad docente como una de las vías para mejorar la calidad educativa general.

Tabla 1. Características de las escuelas eficaces

\begin{tabular}{|l|l|}
\hline \multicolumn{2}{|c|}{ Características de las escuelas eficaces } \\
\hline \multicolumn{2}{|c|}{ Centro } \\
\hline Formación del profesorado. & $\begin{array}{l}\text { Implicación y colaboración de los padres en el proceso de E-A de } \\
\text { los alumnos y en la comunidad. }\end{array}$ \\
\hline Planificación colaboradora. & Interacción Director-Profesores-Alumnos-Padres. \\
\hline Gestión y organización del aula. & “Cultura escolar positiva”. \\
\hline Metodología activa. & Objetivos superiores, claros y concretos. \\
\hline Interacción positiva A/P. & Existencia de buenos lideres institucionales y pedagógicos. \\
\hline Fuerte liderazgo. & $\begin{array}{l}\text { Predominio de la planificación y donde otorgan autonomía a los } \\
\text { profesores. }\end{array}$ \\
\hline $\begin{array}{l}\text { La búsqueda, reconocimiento difusión de } \\
\text { unos valores propios de la escuela. }\end{array}$ & Buen empleo de los recursos humanos. \\
\hline Evaluación del proceso E-A del alumnado. & $\begin{array}{l}\text { Centros que aprovechan el apoyo externo y los recursos } \\
\text { materiales que poseen. }\end{array}$ \\
\hline Evaluación de la práctica docente. & Posibilitan un trabajo efectivo. \\
\hline $\begin{array}{l}\text { Manejo de la heterogeneidad de sus } \\
\text { alumnos. }\end{array}$ & $\begin{array}{l}\text { Desarrollo de actividades con las familias así como un continuo } \\
\text { contacto informativo. }\end{array}$ \\
\hline $\begin{array}{l}\text { Establecer reglas claras y precisas y } \\
\text { fomentar el manejo explícito de la } \\
\text { disciplina. }\end{array}$ & $\begin{array}{l}\text { Promueve el desarrollo de todos los alumnos más allá de lo } \\
\text { esperado. }\end{array}$ \\
\cline { 2 - 2 } & \begin{tabular}{l} 
Coordinación entre el profesorado. \\
\hline
\end{tabular} \\
\hline
\end{tabular}

Fuente: Elaboración propia, basada en Cotton (1995); Edmonds (1982); Purkey \& Smith (1983); \& Ainscow et al., (2001).

\section{Modelos teóricos de escuelas eficaces}

Los primeros estudios teórico-empíricos sobre eficacia escolar, impulsados por la falta en educación de una teoría solida y empíricamente validada, presentan una serie de modelos educativos que recogen una serie de factores agrupados que no podían recibir el calificativo de teorías educativas, pero que contribuyeron al desarrollo de dicha teoría (Murillo, 2008). En este sentido, el modelo de cinco factores de Edmonds (1979) establece que las escuelas eficaces son aquellas que:

\section{Poseen un liderazgo fuerte,}

2. Tienen un clima de altas expectativas en relación al rendimiento de sus alumnos,

3. Presentan una atmósfera ordenada sin ser rígida, tranquila sin ser opresiva,

4. Tienen como objetivo prioritario la adquisición de destrezas y habilidades básicas, y a él se supeditan las actividades del mismo;

5. Realizan una evaluación constante y regular del progreso de los alumnos. 
Por su parte, el modelo de escuelas inusualmente eficaces de Levine y Lezotte (1990), establece unos correlatos claves clasificados en nueve grupos: clima y cultura escolar productivo, atención a la adquisición por parte de los alumnos de las destrezas básicas de aprendizaje, seguimiento adecuado del progreso de los alumnos, desarrollo del profesorado orientado a la práctica y en el lugar de trabajo, liderazgo destacado, fuerte implicación de las familias, organización e implementación instructiva, altas expectativas y exigencias para los alumnos, otros posibles factores. Mientras que el modelo causal de Maureira (2004) se centra principalmente en la relación entre el liderazgo en las organizaciones educativas y la mejora de la eficacia escolar.

Durante los años 90, se continúa reclamando una teoría que explique nuestra realidad educativa y que al mismo tiempo ayude a guiar la labor docente. A partir de teorías instructivas y del aprendizaje, Scheerens y Creemers (1989) reflexionan sobre la necesidad de los modelos comprensivos, que integren elementos claves tanto del centro escolar, del aula y del alumno, modulados por las características de la organización y del contexto, y sus relaciones mutas. Teniendo en cuenta todos estos factores, diseña el modelo integrado de eficacia escolar (Scheerens, 1990,1992), basado en indicadores que aportaban información sobre el funcionamiento de los centros docentes, a nivel de aula y a nivel escolar. Entre los factores de escuela señalan: clima escolar seguro y ordenado, altas expectativas sobre el rendimiento de los alumnos, política escolar orientada al rendimiento, liderazgo educativo, evaluación frecuente del progreso de los alumnos, objetivos claros relativos a las destrezas básicas y consenso y trabajo en equipo entre los profesores. En cuanto a los factores a nivel de aula destacan: tiempo de aprendizaje eficaz o tiempo dedicado a la tarea, enseñanza estructurada o directa, oportunidad para aprender, actitudes y expectativas de los docentes, fomento de la motivación de los estudiantes y alteraciones que el currículum sufre por la situación del hogar de los alumnos.

Igual de importante fue considerado el modelo estadounidense Qait/Macro (Stringfield \& Slavin, 1992) el cual tiene en cuenta los siguientes niveles: el alumno individual, los profesionales con su interacción directa y constante con los alumnos; el centro educativo, con los directivos, el resto del personal del centro y los programas; y por último el nivel superior al centro, que incluye la comunidad, el distrito escolar y el resto de las Autoridades educativas. Dos años después aparece una nueva propuesta, el modelo de eficacia (Creemers, 1994), que aunque contempla los mismos niveles y distribución de diversos factores que el modelo Qait/Macro, lo han convertido en uno los modelos sobre eficacia docente más notables y referenciados hasta el momento (Tabla 2).

Los modelos de comprensión de la eficacia educativa del aula centran la atención en el tiempo, el trabajo en equipo y el comportamiento del profesor, siendo también necesario el análisis del alumnado donde se incluyen sus aptitudes, habilidades, perseverancia, oportunidad y calidad de la enseñanza recibida, así como de la escuela y su contexto, atendiendo especialmente a aquellos factores que afectan directamente al aula. Algunos de los ejes principales que han de incluir según Reynolds (1998) son el tiempo, la oportunidad y la calidad de enseñanza.

Una última propuesta es el modelo de eficacia para centros de Secundaria de Sammons, Thomas y Mortimore (1997), que supone una revisión y ampliación del modelo de Creemers y Scheerens (1994), en el que se ha incluido el nivel del departamento y contempla como una característica más de las escuelas eficaces, la congruencia entre los factores que operan en diferentes niveles (centro, docente, departamento y aula). 
Tabla 2. Modelo de eficacia docente

\begin{tabular}{|c|c|c|}
\hline \multicolumn{3}{|c|}{ Modelo de eficacia docente (Creemers, 1994) } \\
\hline Alumno & \multicolumn{2}{|l|}{$\begin{array}{l}\text {-Motivación } \\
\text {-Antecedentes } \\
\text {-Aptitudes } \\
\text {-Tiempo destinado a la tarea } \\
\text {-Oportunidad para aprender }\end{array}$} \\
\hline Contexto & \multicolumn{2}{|l|}{$\begin{array}{l}\text {-Organización de horarios. } \\
\text {-Supervisión } \\
\text {-Oportunidad de aprender }\end{array}$} \\
\hline \multirow[t]{4}{*}{ Escuela } & Calidad educativa & $\begin{array}{l}\text {-Reglas y acuerdos sobre la enseñanza en el aula. } \\
\text {-Política de evaluación }\end{array}$ \\
\hline & Calidad organizativa & $\begin{array}{l}\text {-Política de coordinación } \\
\text {-Supervisión } \\
\text {-Profesionalidad y cultura escolar orientada a la eficacia. }\end{array}$ \\
\hline & Tiempo & $\begin{array}{l}\text {-Horarios } \\
\text {-Normas } \\
\text {-Acuerdos sobre el empleo del tiempo } \\
\text {-Entorno ordenado y tranquilo }\end{array}$ \\
\hline & Oportunidad para aprender & $\begin{array}{l}\text {-Curriculum escolar/plan de trabajo de la escuela. } \\
\text {-Consenso sobre la misión de la escuela. } \\
\text {-Normas y acuerdos sobre cómo implementar el currículum. }\end{array}$ \\
\hline \multirow[t]{3}{*}{ Aula } & La calidad del curriculum & $\begin{array}{l}\text {-Explicitación } \\
\text {-Ordenación } \\
\text {-Estructuración y claridad de metas y contenidos } \\
\text {-Organizadores previos } \\
\text {-Evaluación }\end{array}$ \\
\hline & Procedimiento de agrupación & $\begin{array}{l}\text {-Agrupación según capacidades } \\
\text {-Aprendizaje cooperativo }\end{array}$ \\
\hline & Comportamiento del profesor & $\begin{array}{l}\text {-Gestión/atmosfera ordenada y tranquila. } \\
\text {-Altas expectativas } \\
\text {-Formulación clara de metas } \\
\text {-Estructuración y claridad en la presentación del contexto. } \\
\text {-Preguntas frecuentes, etc. }\end{array}$ \\
\hline
\end{tabular}

Fuente: Elaboración propia basada en el modelo de eficacia docente de Creemers (1994)

A pesar de la diversidad de modelos existentes sobre escuelas eficaces, todos ellos parten, en primer lugar, del aprendizaje del alumnado, que se basan en el modelo de aprendizaje escolar de Carroll (1963), centrado en cinco elementos básicos: aptitud, habilidad para entender lo que se está enseñando, tenacidad, oportunidad y calidad de la enseñanza (Murillo, 2008); y en segundo lugar, se parte de una visión sistémica del centro educativo, centrada en la interacción entre alumno-aula-escuela y cómo esta interacción contribuye al logro de los alumnos, el clima, el trabajo colegiado de los docentes o el liderazgo (Manzano, de la Iglesia, \& Balcells, 2013).

\section{Papel docente en la gestión eficaz del aula}

En los últimos años el papel de docente se ha ido modificando con el fin de adaptarse a las necesidades educativas promovidas por las transformaciones sociales, actualmente su labor es más complicada y exigente (Carbonel, 2005). Lejos de simplificar su acción educativa, situamos al docente como gestores de los procesos de enseñanza-aprendizaje, lo que implica que todas las dimensiones y factores que se incluyen en las escuelas eficaces pasan necesariamente por la praxis docente. De ahí, la relevancia que tiene el desempeño 
docente en la organización y funcionamiento del centro, pero especialmente en la gestión eficaz del aula.

Dentro del movimiento de escuelas eficaces el tema del docente ha sido vinculado a las competencias. Una escuela eficaz es aquella que se compone de docentes eficaces y esto se traduce en docentes competentes. Ser un profesional de la educación significa contar con conocimientos especializados y recursos variados, que sirvan para gestionar situaciones complejas, comprometido con la tarea, controlando la práctica con autonomía profesional, capaz de transferir y aprender a aprender (Villalobos, 2011).

Una de las competencias fundamentales de los docentes, es formar a los alumnos en función a unas capacidades básicas para que sus alumnos alcancen cuatro aprendizajes esenciales, que en el trascurso de la vida serán para cada persona, los pilares del conocimiento: Aprender a conocer, aprender a hacer, aprender a vivir juntos y aprender a ser, y que les permitirán a enfrentarse a los desafíos que puedan surgir en una sociedad en continuo cambio, razón por la cual surge el concepto de enseñanza por competencias (Delors, 1996; Zabalza \& Arnau, 2007; García \& López, 2011).

Son múltiples las definiciones que podemos encontrar entorno al concepto de competencias, la Comisión Europea la define como una "combinación de destrezas, conocimientos, aptitudes y actitudes adecuadas al contexto, incluyendo la disposición para aprender, y el saber cómo" (CE, 2004, 4-7), por lo que en el espacio Europeo de las Cualificaciones (CCE, 2006) se identifican cuatro elementos de las competencias profesionales: Competencias cognitivas (utilización de teorías y conceptos, así como del conocimiento informal adquirido por la experiencia); Competencia funcional (habilidades y saber hacer); Competencia personal: saber comportarse en las distintas situaciones que se pueden plantear a lo largo del ejercicio profesional; Competencia ética (comportamiento coherente unidos a un conjunto de valores personales y profesionales).

Por otra parte, Escudero (2006), pone el acento en el término 'competencia docente', el cual se asemeja a un grupo de valores, creencias y responsabilidades, sin olvidar los conocimientos técnicos, capacidades y disposición de los docentes, tanto a nivel colectivo, como de esfuerzo personal. Otros autores profundizan en esta perspectiva e identifica una serie de componentes esenciales en las competencias del docente, entre los que cabe destacar la capacidad de integrar todos los conocimientos aprendidos, actuando de acuerdo a las metas propuestas, alcanzando la capacidad de trasferir todo lo aprendido mediante un aprendizaje a lo largo de la vida (Coll \& Martín, 2006).

Sin embargo, Perrenoud (2012) considera que un docente competente debe organizar y dirigir situaciones de aprendizaje, administrar la progresión de los mismos, concebir y hacer evolucionar los dispositivos de diferenciación, envolver a los alumnos en sus aprendizajes y en su trabajo, otorgándoles un papel activo en su propio proceso de E-A, trabajar en equipo, participar en la administración de la escuela, informar y envolver a los padres, hacer uso de las nuevas tecnologías, enfrentar los deberes y los dilemas éticos de la profesión, y administrar su propia formación continua. En este sentido, Escudero (2009) señala que a pesar de que un enfoque por competencias permite tener una visión más constituida de la educación, puede obstaculizar la gestión docente, debido a la complejidad de tener que poner en práctica las competencias propias de su perfil profesional, a la vez que adaptar los contenidos, metodología, y evaluación a las competencias que el educando debe adquirir. En esta línea se presenta a continuación a modo de tabla las distintas competencias que deben adquirir los docentes en la actualidad (Tabla 3). 
Tabla 3. Tipos de competencias del docente

\begin{tabular}{|c|c|c|}
\hline \multicolumn{3}{|c|}{ TIPOS DE COMPETENCIAS DEL DOCENTE } \\
\hline \multirow{3}{*}{$\begin{array}{l}\text { Competencias de } \\
\text { fundamentación } \\
\text { básica }\end{array}$} & Habilidades básicas & $\begin{array}{l}\text { Lectura, redacción, aritmética y matemáticas, expresión y } \\
\text { capacidad de escucha. }\end{array}$ \\
\hline & Aptitudes analíticas & $\begin{array}{l}\text { Capacidad para pensar creativamente, tomar decisiones, } \\
\text { solucionar problemas, procesar y organizar información, saber } \\
\text { aprender y razonar. }\end{array}$ \\
\hline & $\begin{array}{l}\text { Cualidades } \\
\text { personales }\end{array}$ & $\begin{array}{l}\text { Compromiso y autoestima, sociabilidad, gestión personal, } \\
\text { integridad y honestidad. }\end{array}$ \\
\hline \multirow[t]{5}{*}{$\begin{array}{l}\text { Competencias } \\
\text { transversales }\end{array}$} & \multicolumn{2}{|c|}{$\begin{array}{l}\text { Gestión de recursos } \\
\text { Tiempo, dinero, materiales y distribución personal. }\end{array}$} \\
\hline & $\begin{array}{l}\text { Relaciones } \\
\text { interpersonales }\end{array}$ & $\begin{array}{l}\text { Capacidad de participación como miembro de un equipo, } \\
\text { capacidad para enseñar a otros y liderazgo, saber trabajar con } \\
\text { otras personas y negociar. }\end{array}$ \\
\hline & $\begin{array}{l}\text { Gestión } \\
\text { información }\end{array}$ & $\begin{array}{l}\text { Capacidad de búsqueda, organización, interpretación, evaluación } \\
\text { y comunicación de información. }\end{array}$ \\
\hline & $\begin{array}{l}\text { Comprensión } \\
\text { sistémica }\end{array}$ & $\begin{array}{l}\text { Comprende interpretaciones complejas, entiende sistemas, } \\
\text { monitorea y corrige desempeño, mejora o diseña sistemas. }\end{array}$ \\
\hline & $\begin{array}{l}\text { Dominio } \\
\text { tecnológico }\end{array}$ & $\begin{array}{l}\text { Selecciona tecnología en la tarea, da mantenimiento y repara } \\
\text { equipos }\end{array}$ \\
\hline $\begin{array}{l}\text { Competencia para } \\
\text { preguntar }\end{array}$ & \multicolumn{2}{|c|}{ El docente debe establecer si seguir la lógica de la verificación o del descubrimiento. } \\
\hline $\begin{array}{l}\text { Competencias } \\
\text { observacionales }\end{array}$ & \multicolumn{2}{|c|}{$\begin{array}{l}\text { La observación es el primer procedimiento de carácter empírico, la cual se aplica a } \\
\text { cualquier conducta o situación. Por ello es preciso que el docente observe todos los } \\
\text { hechos, acontecimientos y los vaya registrando en un diario o cuaderno de notas. }\end{array}$} \\
\hline $\begin{array}{l}\text { Competencia } \\
\text { analítica }\end{array}$ & \multicolumn{2}{|c|}{ El docente debe tener capacidad para analizar la información. } \\
\hline $\begin{array}{l}\text { Competencia } \\
\text { metodológica }\end{array}$ & \multicolumn{2}{|c|}{ Poseer la capacidad de aplicar procedimientos adecuados. } \\
\hline $\begin{array}{l}\text { Competencia } \\
\text { investigativa }\end{array}$ & \multicolumn{2}{|c|}{$\begin{array}{l}\text { Incluyen competencias para preguntar, observacionales, escriturales, analíticas y las } \\
\text { metodológicas. }\end{array}$} \\
\hline $\begin{array}{l}\text { Competencia } \\
\text { técnica }\end{array}$ & \multicolumn{2}{|c|}{$\begin{array}{l}\text { Capacidad de dominar como experto, la tarea y contenidos de su ámbito de trabajo, así } \\
\text { como, los conocimientos y destrezas necesarias para ello. }\end{array}$} \\
\hline $\begin{array}{l}\text { Competencia } \\
\text { social } \\
\text { participativa }\end{array}$ & \multicolumn{2}{|c|}{$\begin{array}{l}\text { Capacidad para colaborar con otras personas de forma comunicativa y constructiva, y de } \\
\text { decidir y asumir responsabilidades. }\end{array}$} \\
\hline
\end{tabular}

Fuente: Elaboración propia basada en Mertens (2000) \& Bunk (1994)

Braslavsky (1999) afirma que la clave para promover la reinvención de la labor de la educación está en desarrollar competencias para un mejor desempeño y para una mejor participación en reinvención de la escuela y de los sistemas educativos. Estas competencias se relacionan con conocimientos ubicados dentro de un curriculum, transmitido por formadores.

\section{Metodología}

\section{Objetivos}

El objetivo principal de este estudio es conocer y comparar las distintas formas de gestionar el aula que emplean los profesores noveles y con experiencia en grupos aula de similares características. En cuanto a los objetivos específicos, estos son clasificados de la siguiente manera:

- Identificar la tendencia a una planificación coordinada interprofesionales o individual. 
- Conocer la metodología utilizada por cada uno de los profesionales.

- Conocer las distintas estrategias para resolver conflictos en el aula.

- Reconocer la frecuencia con la que se mantienen relaciones interpersonales con el profesorado, alumnado y familiares.

Detectar el tipo de evaluación que llevan a cabo ambos profesionales.

\section{Instrumentos}

Para la recogida de información se utilizaron dos tipos de instrumentos de elaboración propia sometidos a un proceso de validación mediante la técnica Delphi. El primero de los instrumentos se trata de un cuestionario "De gestión docente", que permite conocer aspectos sobre Planificación, coordinación interprofesional, metodología, resolución de conflictos, la relación entre alumno-profesor-familia y la evaluación. El segundo instrumento, se corresponde a la parrilla de observación "Sobre gestión del aula", en la que se recogen diferentes indicadores de observación agrupados en tres categorías diferenciadas entre sí: planificación, resolución de conflictos y metodología. Para la aplicación de este instrumento, se estableció un periodo de observación de dos semanas por profesor, donde cada docente era observado a diario. La parrilla de observación permitió ampliar, verificar o refutar la información ofrecida por el cuestionario.

\section{Análisis de resultados}

Para la realización de este estudio hemos recurrido a un centro educativo de educación Infantil y Primaria de la población de Cieza, localidad de Murcia. La elección de este centro obedece a la necesidad de tener un profesor/a novel con menos de 2 años de experiencia y un profesor/a experta con más de 8 años de experiencia en cada uno de los grupos de un mismo nivel aula, por tanto debía ser un centro con al menos dos líneas. Concretamente se localizaron a cuatro profesoras, dos con menos de 2 años de experiencia y 2 con más de 8 , que comparten clase en el primer ciclo de primaria $\left(1^{\circ} \mathrm{A}\right.$ y $\left.1^{\circ} \mathrm{B}\right)$ y segundo ciclo de primaria $\left(2^{\circ}\right.$ A y $2^{\circ} \mathrm{B}$ ), impartiendo las mismas asignaturas.

Los datos que se exponen a continuación responden al análisis de los resultados obtenidos del cuestionario y de la observación directa. Del análisis de cuestionarios se puede comprobar que, la totalidad de los docentes (100\%) aseguran realizar un trabajo previo a la planificación, las noveles (50\%) diseñan unidades didácticas, y los expertos (50\%) además establecen una batería de actividades previas y planifican actividades de apoyo y atención a la diversidad. En el momento de la planificación a las profesoras expertas (50\%) no les supone ningún esfuerzo elaborar actividades acordes a los contenidos, elaborar material adicional, planificar con la colaboración de otros profesionales y establecer estrategias de evaluación, sin embargo a los noveles (50\%) lo que más esfuerzo le supone es elaborar material adicional para el alumnado.

A la hora de planificar tienen en cuenta la atención a la diversidad (75\%), los noveles (50\%) realizan adaptaciones en los contenidos y en el tiempo, aunque los expertos (50\%) también adaptan la metodología, evaluación y espacios, considerando necesario poseer los recursos humanos necesario y elaborar actividades de apoyo o refuerzo.

En lo que respecta a la evaluación tanto los noveles como los expertos (100\%) considera imprescindible la evaluación continua, como estrategia de progreso y para ajustar los procesos educativos a los objetivos establecidos. Reconocen que las bajas calificación son debidas, en el caso de los noveles (50\%) a la falta de dedicación a los estudios por parte de los alumnos, de realización de tareas diarias y de motivación, mientras que los expertos (50\%) apuntan a la falta de implicación de los padres en el proceso de E-A de sus hijos, la 
falta de motivación y realización de tareas diarias, como motivos principales de los malos resultados académicos de sus alumnos.

Ambas docentes (100\%) manifiestan tener conocimientos sobre sus alumnos: los noveles (50\%) sobre problemas de salud y la situación personal de las familias, mientras que los expertos (50\%) además sobre el barrio en el que viven y el entorno que les rodea. Tanto noveles como expertas tienen contacto frecuente con las familias (100\%), a través de reuniones trimestrales obligatorias, mientras que los noveles (50\%) recurren también al contacto diario y los expertos a las notas para casa (50\%).

Los datos que se muestran a continuación parten de un total de 40 observaciones realizadas en el aula, que corresponde al 100\% de los datos totales. A cada uno de los docentes se le asigno un total de 10 observaciones (25\%). A continuación se muestran los datos obtenidos del estudio comparativo entre docentes noveles y expertos. En lo que respecta al análisis de la observación directa, cada indicador objeto de observación ha sido considerado como una variable dicótoma, cuyas opciones de respuesta corresponden a: 1. Si tienen lugar en el aula, y 2. No tienen lugar en el aula, en el momento de la observación. Tras las observaciones directas de las prácticas docentes se concluye que: metodológicamente los noveles recurren al aprendizaje basado en tareas (80\%), aprendizaje colaborativo (40\%), clases magistrales participativas (70\%), juegos, mapas, canciones, salidas al exterior y libro de texto (80-90\%), mientras que los expertos se centran en el aprendizaje basado en tareas (90-100\%) y el libro de texto (70-90\%) como recurso (Gráfica 2).

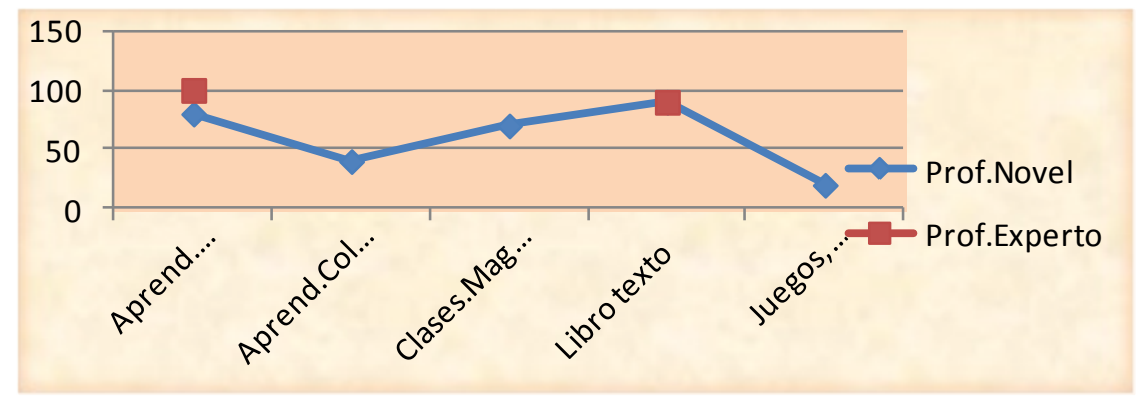

Gráfica 2: Metodología empleada por Noveles y Expertos

Para despertar la motivación en el alumnado, ambas recurren al reconocimiento (80-100\%), aunque las noveles también lo hacen mediante el reconocimiento social (70-90\%) y los expertos a través de las llamadas de atención (50-60\%). Para recuperar el orden, los expertos recurren al castigo (30\%), y los noveles alzando la voz (30\%) (Gráfica 3).



Gráfica 3: Técnicas para recuperar el orden 
Ambos perfiles, facilitan un clima de confianza en el alumnado(100\%), lanzan preguntas de reflexión y debate, ofrecen apoyo a todos los alumnos, fomentan la participación del alumnado (70-90\%), realizan una valoración posterior de las actividades realizadas (60-70\%), sin embargo no solicitan la opinión de los educando en la toma de decisiones sobre contenidos, actividades y temporalización (100\%), no adquieren la función de mediador , ni orientador-dinamizador (60-70\%), no promueven la toma de decisiones por consenso (70$100 \%$ ), no cuentan con un diseño del proceso de E-A creativo y formador (40-50), y no utilizan las dinámicas de grupo (80\%) (Gráfica 4).



Gráfica 4: Gestión del aula por Noveles y Expertos

Además los expertos no favorecen el trabajo en equipo, no fomentan la autonomía del alumnado (90-100\%), ni facilitan la capacidad de aprender sin depender del libro ni del profesor (60-80\%). Los noveles facilitan tiempo para que los alumnos reflexionen sobre lo aprendido, y trabajan en agrupaciones grandes (70\%) aunque también lo hacen frecuentemente mediante el trabajo individualizado (90-100\%), pero no favorecen la ayuda mutua entre compañeros (60-70\%). Por el contrario los expertos no ofrecen tiempo de reflexión al alumnado (20\%), basan su práctica docente en el trabajo individual (95\%) y no fomentan la ayuda mutua entre compañeros (10\%) (Gráfica 5).

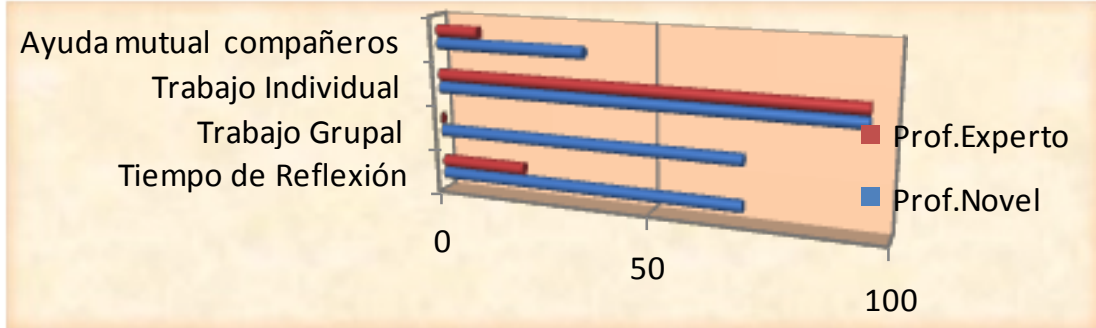

Gráfica 5: Autonomía y trabajo en equipo

En relación a la resolución de conflictos en el aula, en el caso de los noveles éstos surgen sobre todo durante el momento del trabajo del alumnado (50\%) y en el de los expertos, al comienzo de las sesiones (25-50\%). Tanto en un caso como en otro, el conflicto es de relación- comunicación (50-100\%) y son gestionados individualmente (83,3-100\%), aunque los noveles en ocasiones recurren a los alumnos (14,3-16,7\%). Ambos perfiles gestionan el conflicto a través de conductas democrático-asertivas y mediante el diálogo (83,3-100\%), aunque los noveles en ocasiones acuden a las llamadas de atención (22,6- 33,3\%), excepcionalmente a conductas violentas $(14,3-16 \%)$ y los expertos recurren al castigo (80100\%) (Gráfica 6). 


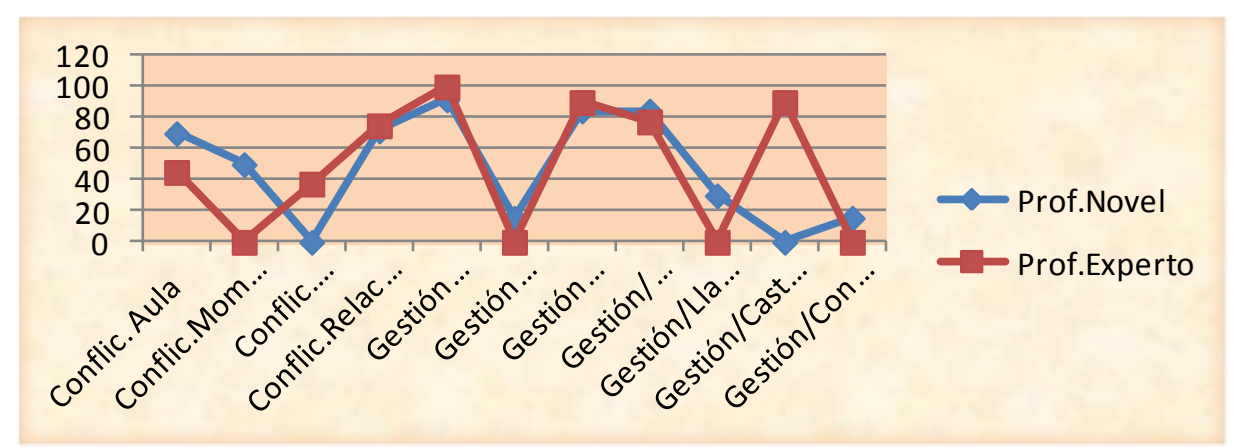

Gráfica 6: Resolución de conflictos

Como estrategias de presentación de sus sesiones, las profesoras expertas establecen actividades de inicio $(60 \%)$ y transición $(80 \%)$ y proponen una parte teórico $(50-60 \%)$-práctica (90-100\%), por el contrario las noveles recurren a las actividades de transición (90\%) y proponen una parte práctica (100\%) (Gráfica 7).

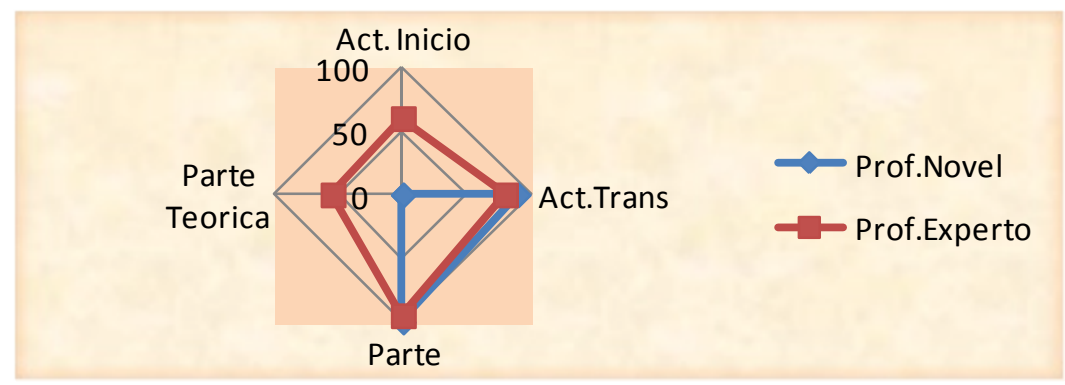

Gráfica 7: Estrategias de presentación de sesiones

\section{Discusión y conclusiones}

A partir de los datos extraídos de los informes anteriores, se podría afirmar que a la hora de planificar, los expertos consideran necesario abarcar ciertos aspectos que los noveles dejan en el olvido, como la elaboración de materiales y recursos, el estudio de casos individuales, seguimiento y evaluación del proceso E-A, resolución de conflictos, planteamiento de contenidos, actividades, metodología y evaluación y atención a la diversidad. Otros estudios revelan que los docentes en la planificación dedican especial atención a la Atención a la diversidad, concretamente el $83 \%$ se basan en las experiencias y conocimientos previos que traen los alumnos y respetan los intereses y necesidades de éstos en el momento de planificar actividades (Pérez, 2003).

Metodológicamente los noveles fomentan el trabajo en equipo, práctica que no utilizan los expertos, aunque en ambos casos hacen uso del aprendizaje basado en tareas, y el libro de texto. Aunque somos conscientes que la controversia y polémica del trabajo en equipo frente al individual, metodología tradicional frente a metodologías activas está presente, compartimos con Zabalza (2011) que en educación es necesario una metodología mixta complementada con aspectos básicos de métodos clásicos como lecciones magistrales, el trabajo en equipo y el trabajo independiente de los estudiantes. 
Respecto a las estrategias utilizadas por los docentes para despertar la motivación del alumnado, en ambos casos se recurre al reconocimiento, aunque los expertos también hacen uso del reconocimiento social y los noveles de las llamadas de atención.

En lo que se refiere a la relación con las familias los noveles mantienen relaciones más directas y constantes con las familias de sus alumnos, mientras que los expertos lo hacen a través de técnicas que requieren menos contacto físico y comunicación verbal. En otras investigaciones se ha podido comprobar que la vía de comunicación más habitual entre familias y escuela son las entrevistas puntuales con el tutor, seguido de las reuniones grupales con éste, del horario de tutoría y de los contactos casuales con el tutor, mientras que los procedimientos más utilizados para realizar las citas entre profesores y padres son a través de cartas o de la agenda (García, Hernández et al., 2010). En cuanto a la relación que mantienen con el alumnado, en ambos casos fomentan unas relaciones favorables basadas en un clima de cordialidad y cercanía, fundado en el respeto mutuo y en un trato cercano entre A-P, donde se proporciona confianza para expresar ideas, opiniones y dudas.

Finalmente, en cuanto a la resolución de conflictos, las técnicas de gestión democráticodialogantes se muestran con mayor presencia en los noveles a la hora de abordar los conflictos en el aula. Estos resultados nos han permitido comprobar, entre otros aspectos, la importancia de una gestión eficaz del aula en la creación de condiciones que faciliten los procesos de enseñanza-aprendizaje y que mejoren la convivencia en los centros; así como la necesidad de reflexionar sobre el rol que el docente desempeña y sobre las competencias básicas profesionales necesarias para llevar a cabo una eficaz gestión de aula. Con nuestra investigación esperamos contribuir a la identificación de los perfiles de los docentes eficaces y a la elaboración de nuevas propuestas de formación de profesorado.

\section{Bibliografía}

Ainscow, M., Hopkins, D., Southworth, G., \& West, M. (2001). Hacia escuelas eficaces para todos. Manual para la formación de equipos docentes. Madrid: Narcea.

Beltrán, F. (2007). Política versus gestión escolar. Revista Novedades Educativas, 18, 4-8

Braslavsky, C. (1999). Bases, orientaciones y criterios para el diseño de programas de formación de profesores. RIEOEI, 19.

Bunk, GP. (1994). La transmisión de las competencias en la formación y perfeccionamiento profesional de la RFA. Revista europea formación profesional 1, 8-14.

Carbonell, J. (2005). Un largo camino. Treinta años de historia. Cuadernos de Pedagogía, 342, 48-53.

Carroll, J.B. (1963). A model of school learning. Teachers College Record, 64, 723-733.

Castro, A. (2012). La gestión escolar en cuestión. Cuadernos de Educación, (4), 225-233.

Coll, C., \& Martín, E. (2006). Vigencia del debate curricular: aprendizajes básicos, competencias y estándares. Revista PRELAC, 3, 6-27.

Consejo de la Unión Europea. (2006). Recomendación del parlamento europeo y del consejo de 18 de diciembre de 2006 sobre las competencias clave para el aprendizaje permanente (2006/962/CE).

Cotton, K. (1995). Effective schooling practices: A research synthesis. 1995 updated. Portland, OR: Northwest Regional Educational Laboratory. 
Creemers, B. (1994). The effective classroom. London: Cassell

Delors, J. (1996). La educación encierra un Tesoro. Informe a la UNESCO de la Comisión Internacional sobre la Educación para el Siglo XXI. Ediciones UNESCO.

Edmonds, R. E. (1982). Programs of school improvement: An overview. Educational Leadership, 40, 4-11.

Edmonds, R.R. (1979). Effective schools for the urban poor. Educational Leadership, 40 (3), 4-11.

Escudero, J. M. (2009). La formación del profesorado de Educación Secundaria: contenidos y aprendizajes docentes. Revista de educación, (350), 79-103.

Escudero, J.M. (2006). La formación del profesorado y la mejora de la educación ( ${ }^{\mathrm{a}}$ ed.). Ediciones Octaedro. España.

Fullan, M. (1996). Leadership for change. In International handbook of educational leadership and administration, 701-722. Springer Netherlands.

García, L \& López, R. (2011). Convivir en la escuela. Una propuesta para su aprendizaje por competencias. Revista de educación, 356, 531-555.

García, M.P., Gomariz, M.Á., Hernández, M.Á., \& Parra, J. (2010). La comunicación entre la familia y el centro educativo, desde la percepción de los padres y madres de los alumnos. Educatio Siglo XXI, 28 (1), 157-188.

Gómez, R.L. (2010). Calidad Educativa: Más que resultados en pruebas estandarizadas. Revista Educación y Pedagogía, 16(38).

González, J.L. (2005). De la gestión pedagógica a la gestión educativa. Una tarea inconclusa. Observatorio Ciudadano de la Educación. Colaboraciones Libres. V, (185). Graó.

González, R. P. M., Vázquez, E. B., \& Rodríguez, E. J. M. (2013). El liderazgo educativo. Consideraciones generales. Revista Didasc@lia: Didáctica y Educación 4(1), 165-180.

Lavín, S. (2007). Transitando desde la gestión de un establecimiento hacia la gestión de un centro de desarrollo educativo. Educare, 2, 23-35.

Levine, D. U., \& Lezotte, L. W. (1990). Unusually Effective Schools: A Review and Analysis of Research and Practice. Madison: National Center for Effective Schools Research and Development.

Manzano, J. M., de la Iglesia, J. M. M., \& Balcells, J. C. (2013). La planificación estratégica, un indicador sobre el liderazgo pedagógico. Teoría de la Educación. Educación y Cultura en la Sociedad de la Información, 14(2), 292-315.

Martínez, D., Collazo, M., \& Liss, M. (2009). Dimensiones del trabajo docente: una propuesta de abordaje del malestar y el sufrimiento psíquico de los docentes en la Argentina. Edu. Soc., Campinas 107, (30), 389-408.

Maureira, O. (2004). El liderazgo factor de eficacia escolar, hacia un modelo casual. REICE, 2 (1), 1-20.

Mertens, L. (2000). La gestión por competencia en la empresa y la formación profesional, OEl, España (Internet).

Muñoz, J. M. E. (2009). Las competencias profesionales y la formación universitaria: posibilidades y riesgos. Pedagogía social. Revista interuniversitaria, (16), 65-82. 
Murillo, F. J. (2008). Hacia un modelo de eficacia escolar. Estudio multinivel sobre los factores de eficacia en las escuelas españolas. Revista electrónica iberoamericana sobre calidad, eficacia y cambio en educación, 6 (1), 4-28.

Murillo, F.J. (2003). Una panorámica de la investigación iberoamericana sobre eficacia escolar. Revista electrónica iberoamericana sobre calidad, eficacia y cambio en educación, 1 (1).

Murillo, F.J. (2005). La investigación sobre eficacia escolar. Barcelona: Octaedro.

Ortega, F.J. (2008). Tendencias en la gestión de centros educativos. Revista Latinoamericana de Estudios Educativos. XXXVIII (1-2), 61-79.

Pérez, A. (2003). Actitud del docente ante la puesta en práctica del proyecto pedagógico de aula en la I etapa de educación básica en la Universidad educativa estadal "Lucia Palacios". (Trabajo de grado) Universidad Nacional abierta. Republica Bolivariana de Venezuela.

Perrenoud, P. (2012). Cuando la escuela pretende preparar para la vida: ¿desarrollar competencias o enseñar otros saberes? (Vol. 40). Grao.

PISA (2012). Programa para la evaluación internacional de los alumnos: OCDE: informe español 1. Resultados y contexto. Madrid 2013.

Purkey, S. C., \& Smith, M. S. (1983). Effective Schools. The Elementary School Journal, 83, 412452.

Reynolds, D., Bollen, R., Creemers, B., Hopkins, D., Stoll, L., \& Lagerweij, N. (1996). Las escuelas eficaces: claves para mejorar la enseñanza. Santillana.

Sammons, P., Thomas, S., \& Mortimore, P. (1997). Forging links: Effective schools and effective departments. Sage.

Scheerens, J. (1992). Effective Schooling Research: Theory and Practice. New York: Cassell.

Stringfield, S., \& Slavin, R. (1992). A hierarquical longitudinal model for elementary school effects. En B. Creemers y G.J. Reezigt (Eds.), Evaluation of educational research, 3569. Groningen: ICO.

Villalobos, X. (2011). Reflexión en torno a la gestión de aula y a la mejora en los procesos de enseñanza y aprendizajes. Revista Iberoamericana de educación, 55, 1-7.

Zabala, A., \& Arnau, L. (2007). 11 ideas clave, como aprender y enseñar competencias. Barcelona: Graó.

Zabalza, M.A. (2011). Metodología docente. Teaching Methodology. Revista de docencia universitaria. 9 (3), 75-98. 


\section{Autores}

Antonia Penalva López

Titulada en Educación Social, Pedagogía, Máster de innovación e investigación en educación infantil y primaria, y Doctoranda. Autora de publicaciones como: Práctica docente. Estudio de casos a través de la observación directa; Convivencia escolar como reto educativo. Programas de intervención; Gestión del Aula. Una cuestión de Responsabilidad Docente; Análisis comparativo de las competencias profesionales del profesor novel y experto de primaria (2012); Una apuesta por la resolución de conflictos. Análisis de los planes de convivencia; La gestión del conflicto como mejora de la convivencia escolar. Estudio de casos; Percepción de los expertos de la necesidad de la formación del profesorado en convivencia (2013)

Ma Ángeles Hernández Prados

Profesora de la Facultad de Educación de la Universidad de Murcia. Mi producción científica se enmarca en las siguientes líneas de investigación: Educación para la ciudadanía y mejora de la convivencia escolar (Formación del profesorado, escuelas de padres, etc.), Educación en valores en el contexto familiar (Resolución de conflictos, aprendizaje de normas, implicación parental en las cuestiones escolares, educar en y desde la responsabilidad, etc.), y Perspectiva ético-moral de la Sociedad de la Información (la seguridad de los menores en la red, ciudadanía digital, cyberbullying, etc.)

\section{Catalina Guerrero Romera}

Profesora de la Facultad de Educación de la Universidad de Murcia. Coordinadora de Formación Corporativa del Vicerrectorado de Desarrollo Estratégico y Formación de dicha Universidad. Consultora de Formación y Desarrollo Organizativo. Sus líneas de investigación actuales, algunas publicadas en diversas revistas de especialización científica, están relacionadas con la Formación, Evaluación, Innovación y Mejora en las Organizaciones, la Gestión del Conocimiento y el Aprendizaje Organizacional y la Orientación Profesional y Laboral 